\title{
Surface Integrated Printed Interdigital Structure for Process Monitoring the Curing of an Adhesive Joint ${ }^{\dagger}$
}

\author{
Martina Hübner *, Walter Lang and Gerrit Dumstorff \\ Institute for Microsensors, -Actuators and -Systems (IMSAS), Microsystems Center Bremen (MCB), \\ University of Bremen, Bremen, Germany; wlang@imsas.uni-bremen.de (W.L.); \\ gdumstorff@imsas.uni-bremen.de (G.D.) \\ * Correspondence: mhuebner@imsas.uni-bremen.de; Tel.: +49-421-218-62618 \\ + Presented at the Eurosensors 2017 Conference, Paris, France, 3-6 September 2017.
}

Published: 11 August 2017

\begin{abstract}
We present a printable interdigital structure, integrated into the surface of a component, to monitor the curing of two adhesively joint components during the fabrication process. In the factory of the future there is a need of new sensor concepts beside the information technological infrastructure. Monitoring of the fabrication enables automated control of the process which improves the quality of adhesive joints. In addition, the cost can be reduced by individually adapted curing times. We demonstrate that cure monitoring with the printed interdigital structure is possible. Two adhesives, a fast and a slow curing one, are analyzed to show the high potential of this process monitoring method.
\end{abstract}

Keywords: printed sensor; interdigital structure; cure monitoring; impedance spectroscopy; factory of the future

\section{Introduction}

Adhesives are used in a huge variety of applications to join components such as sheet metal, plastic or wooden parts. Different approaches based on optical [1], acoustic [2] or impedance measurements [3] to qualify the adhesive joint have been developed over the last decades. The most common is impedance spectroscopy, where an interdigital structure on a flexible or rigid substrate is evaluated [4,5]. The adhesive is placed on top of the sensor structure or the sensor structure itself is placed in the adhesive. During curing, the ion viscosity of the polymer changes which leads to a change in the dielectric properties of the polymer.

In the factory of the future all processes are based on computer integrated manufacturing. This will lead to two main advantages: cost efficiency and higher flexibility [3]. Today, the main focus towards the factory of the future is based on software (e.g., cloud-based computing) and the infrastructure, to link machines with machines and a global control system (information technology infrastructure). Beside the software and the infrastructure, there is a need of new hardware, mainly sensors and sensor concepts, to get precise online data from the manufacturing process itself. This is necessary to ensure quality assurance and to optimize manufacturing. Our idea is the integration of a printed interdigital structure in the surface as shown in Figure 1. After applying adhesive (Figure 1b) the two components are joined. During curing process, the sensor is evaluated by the central control system as seen in Figure 1c. At the point of sufficient curing this new set-up part can be further processed. While curing processes strongly depends on the environmental conditions, the central control system can individually adapt curing times and determine which parts are finished for further processing. Thus, we get an energy efficient and controlled curing process with an autonomous decision when curing cycle is finished. 
(a) Component 1 with surface integrated printed interdigital sensor

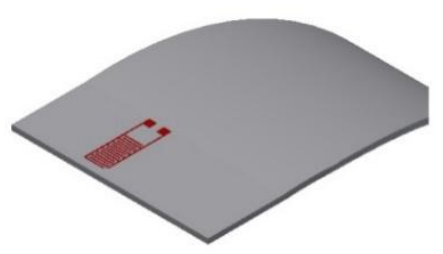

(b) Applying Adhesive

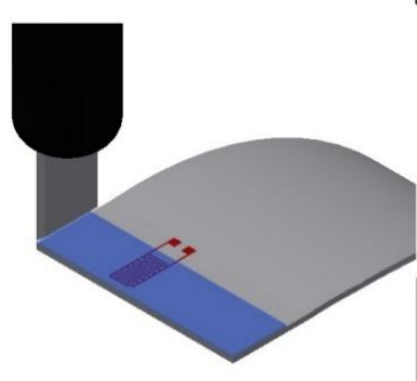

(c) Joining Component 2 with Component 1 and monitoring the curing process

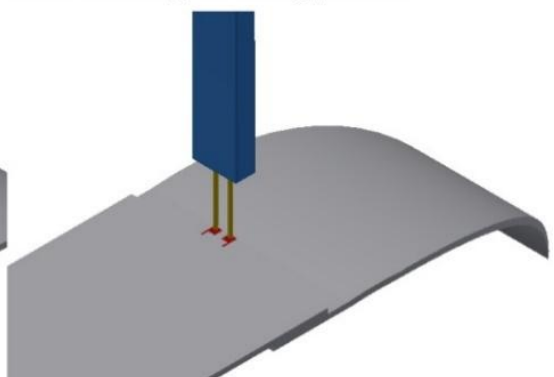

Figure 1. Schematic automated fabrication process with integrated interdigital structure for cure monitoring.

\section{Experimental Setup}

The layout of the sensor is shown in Figure 2a. It consists of an interdigital structure of two times ten fingers, having a width of $w_{\text {finger }}=200 \mu \mathrm{m}$ and a distance of $d_{\text {finger }}=400 \mu \mathrm{m}$. The sensor is printed on metallic aluminum sheet, which is joined adhesively with another aluminum sheet of same size. The adhered surface is $A=20 \times 20 \mathrm{~mm}^{2}$.

(a) layout

(dimensions in $\mathrm{mm}$ )

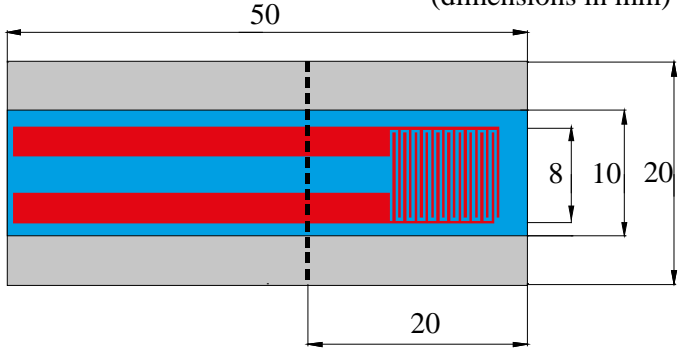

(b) process flow

1. printing insulation $(10 \mu \mathrm{m})$ on aluminum sheet $(1.5 \mathrm{~mm})$

2. printing interdigital structure

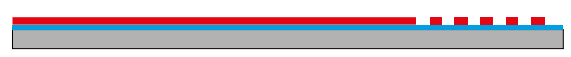

3. Applying adhesive and joining aluminum sheets

Figure 2. Sensor layout and process flow of the printed interdigital structure on aluminium sheets.

Figure $2 \mathrm{~b}$ shows the fabrication of the sensor and the process of joining the two metallic sheets. In the first step insulation (two-component epoxy) is printed on the sheet. This is followed by screen printing the interdigital sensor structure. For this the silver paste DuPont Kapton KA801 is used. This paste consists of polyimide as polymer binder. Thus, a high chemical resistance is achieved and the sensor does not get chemically destroyed due to the adhesive which is finally monitored. The printed part is then joined with an uncovered metal part. The tests are done with two different solvent free two-component epoxy mixture. While the binder material of both adhesives is based on bisphenol, the hardeners are different: one is slow curing type based on amines (UHU Plus Endfest) and the other one is of fast curing type, based on polymercaptan (UHU Plus Schnellfest). The sensor sheet and the joined sheets are shown in Figure 3. During the curing process, the impedance of the interdigital structure is measured. 


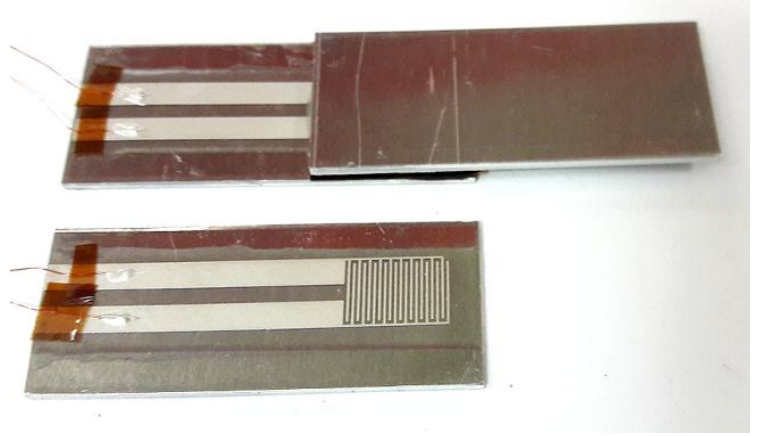

Figure 3. Screen printed sensor on metal part to be joint. The interdigital structure consist of $\mathrm{N}=20$ fingers.

\section{Measurement Results and Discussion}

In Figure 4a the absolute value of the impedance during curing of the fast curing epoxy is plotted in a frequency range from $f=1 \mathrm{~Hz}$ to $1 \mathrm{kHz}$. After applying the adhesive the viscosity increases. The absolute value of the impedance increases due to the crosslinking of the adhesive. The movement of the free charges is reduced and the resistance increases. This is the main reason for the impedance increase which can be therefore used as a monitoring method. After twelve hours, curing is completed. No changes in the impedance can be seen, which correlates with the manufacturers specifications. However, in many industrial processes adhesives with shorter curing times are used to reduce processing times. Therefore, we choose a fast curing adhesive (UHU Plus Schnellfest), curing in less than $t=20 \mathrm{~min}$ at room temperature (according to the datasheet). In this case we measure in a smaller frequency range to reduce the measuring time of one frequency sweep. Figure $4 \mathrm{~b}$ shows the impedance measurement over time. The measurement directly starts after applying the adhesive and ends with the fully cured adhesive. After $t=8 \mathrm{~min}$ the main part of the adhesive is crosslinked. This demonstrates the potential to reduce the curing time depending on the measured impedance. While the manufacturer of the adhesive might give more conservative values for the curing time where curing is definitely sufficient, lower cycle period can be achieved by online monitoring.

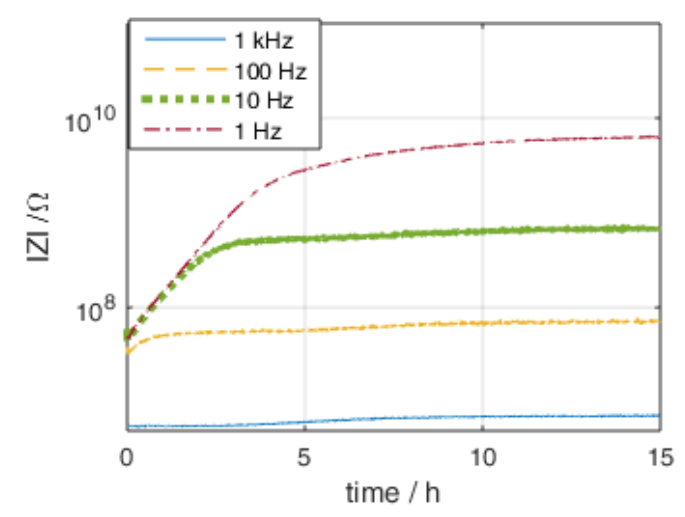

(a)

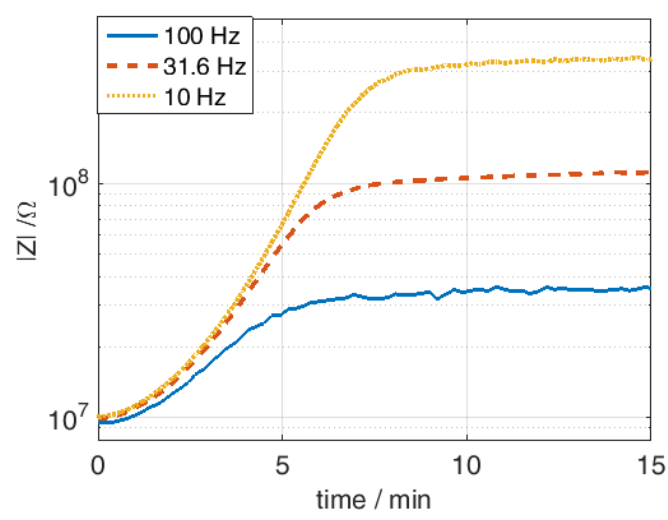

(b)

Figure 4. Absolute value of the impedance during curing. The measurement is started directly after applying the adhesive. (a) slow curing two-component epoxy; (b) fast curing two-component epoxy.

\section{Conclusions and Outlook}

We have presented a printed interdigital structure to monitor the curing of different adhesives during joining of two metallic sheets. Our concept shows the simplicity of the printed interdigital structure and its potential of monitoring curing of adhesives in factories of the future. The structure can easily be integrated in any component. By evaluating the degree of curing by impedance 
spectroscopy, the process of joining components with adhesives can be evaluated and possibly controlled by a central control system.

In future work, adhesives with different curing mechanism, like polycondensation (e.g., polyimide or silicone) or polymerization adhesives (e.g., cyanoacrylate or unsaturated polyesters) should be investigated. Furthermore, the sensor itself might reduce the adhesion of the adhesive. Due to this, the mechanical structure can be weakened which is called "the foreign body effect" [7]. Mechanical tests will be done to investigate the influence of the sensor on the mechanical strength of the bonding. In addition to different sensor designs and adhesives, also adhesion promoters can be tested and evaluated, to bring the foreign body effect to a minimum.

Acknowledgments: The work is funded by the German Research Foundation (DFG) within the project "Kammsensor: Integration flexibler kapazitiver Sensoren in Polymere zur Überwachung von Polymerisationsund Degradationsprozessen" (LA 1471/17-1) and by the University of Bremen within the postdoctoral research project "AddSense: Additive manufacturing for local fabrication of sensor structures for material integrated sensing".

Conflicts of Interest: The authors declare no conflict of interest. The founding sponsors had no role in the design of the study; in the collection, analyses, or interpretation of data; in the writing of the manuscript, and in the decision to publish the results.

\section{References}

1. Murukeshan, V.M.; Chan, P.Y.; Ong, L.S.; Seah, L.K. Cure monitoring of smart composites using fiber Bragg grating based embedded sensors. Sens. Actuators A Phys. 2000, 79, 153-161.

2. Freemantle, R.J.; Challis, R.E. Combined compression and shear wave ultrasonic measurements on curing adhesive. Meas. Sci. Technol. 1998, 9, 1291.

3. Habenicht, G. Kleben: Grundlagen, Technologie, Anwendungen; Springer: Berlin/Heidelberg, Germany; New York, NY, USA, 1997; Volume 3.

4. Yang, Y.; Chiesura, G.; Vervust, T.; Bossuyt, F.; Luyckx, G.; Degrieck, J.; Vanfleteren, J. Design and fabrication of a flexible dielectric sensor system for in situ and real-time production monitoring of glass fibre reinforced composites. Sens. Actuators A 2016, 243, 103-110.

5. Kim, H.G. Dielectric cure monitoring for glass/polyester prepreg composites. Compos. Struct. 2002, 57, 91-99.

6. Scheer, A.-W. CIM Computer Integrated Manufacturing: Towards the Factory of the Future; Springer Science \& Business Media: New York, NY, USA, 2012.

7. Dumstorff, G.; Paul, S.; Lang, W. Integration without disruption: The basic challenge of sensor integration. IEEE Sens. J. 2014, 14, 2102-2111, doi:10.1109/JSEN.2013.2294626. 